UDC: 530.182

\title{
Electric field effects in chemical patterns
}

\author{
P. Dähmlow ${ }^{1, a}$, Ch. Luengviria ${ }^{2}$, S. C. Müller ${ }^{1, b}$ \\ ${ }^{1}$ Institute of Experimental Physics, University Magdeburg, Universitätsplatz 2, D-39106 Magdeburg, Germany \\ ${ }^{2}$ Department of Physics, Kasetsart University, 50 Phaholyothin Road, Jatujak, Bangkok 10900, Thailand \\ E-mail: ${ }^{a}$ patricia.daehmlow@ovgu.de, ${ }^{\mathrm{b}}$ stefan.mueller@ovgu.de
}

Received June 6, 2014

Excitation waves are a prototype of self-organized dynamic patterns in non-equilibrium systems. They develop their own intrinsic dynamics resulting in travelling waves of various forms and shapes. Prominent examples are rotating spirals and scroll waves. It is an interesting and challenging task to find ways to control their behavior by applying external signals, upon which these propagating waves react. We apply external electric fields to such waves in the excitable Belousov-Zhabotinsky (BZ) reaction. Remarkable effects include the change of wave speed, reversal of propagation direction, annihilation of counter-rotating spiral waves and reorientation of scroll wave filaments. These effects can be explained in numerical simulations, where the negatively charged inhibitor bromide plays an essential role. Electric field effects have also been investigated in biological excitable media such as the social amoebae Dictyostelium discoideum. Quite recently we have started to investigate electric field effect in the BZ reaction dissolved in an Aerosol OT water-in-oil microemulsion. A drift of complex patterns can be observed, and also the viscosity and electric conductivity change. We discuss the assumption that this system can act as a model for long range communication between neurons.

Keywords: autowaves, chemical patterns, electric field effects

\section{Эффекты воздействия электрического поля на химические структуры}

\author{
П. Демлов ${ }^{1}$, Х. Люнгфириа ${ }^{2}$ ШІ. К. Мюллер ${ }^{1}$ \\ ${ }^{1}$ Магдебургский университет, Институт экспериментальной физики, \\ Германия, Магдебург D-39106, Униферзитетсплаи, 2 \\ ${ }^{2}$ Университет Касетсарт, отделение физики, Тайланд, Бангкок, 10900, Чатучак, Фахолиотин Роад, 50
}

Волны возбуждения являются прообразом самоорганизующихся динамических структур в неравновесных системах. Они характеризуются своей собственной внутренней динамикой, приводящей к формированию бегущих волн различных типов и форм. Яркие примеры - это вращающиеся спирали и скрученные свитки. Интересная и сложная задача - найти способы управления их поведением, применяя внешние сигналы, влияющие на распространяющиеся волны. В качестве такого воздействия мы используем внешние электрические поля, наложенные на возбудимую реакцию Белоусова-Жаботинского (БЖ). Существенные эффекты влияния полей на волны включают изменение скорости волны, обращение направления распространения, взаимное уничтожение вращающихся в противоположных направлениях спиральных волн и переориентацию нитей скрученных свитков. Эти эффекты могут быть объяснены в численных экспериментах, при этом существенную роль играет отрицательно заряженный ингибитор бромид. Эффекты электрического поля также были исследованы в биологических возбудимых средах, таких как социальные амебы Dictyostelium discoideum. Совсем недавно мы начали исследовать влияние электрического поля на реакцию БЖ, протекающую в водно-масляной микроэмульсии. Удалось наблюдать дрейф сложных структур, а также изменение вязкости и электрической проводимости. Мы обсуждаем предположение, что эта система может выступать в качестве модели для дальнодействующего взаимодействия между нейронами.

Ключевые слова: автоволны, структуры в химических системах, действие электрических полей Citation: Computer Research and Modeling, 2014, vol. 6, no. 5, pp. 705-718. 


\section{Introduction}

Pattern formation is ubiquitous in nature. We all observe this phenomenon on a daily basis. These patterns assume a plethora of forms and shapes, which may or may not change in time on a fast or a slow time scale. They occur in the inanimate nature and in living systems, where they may be involved in evolutionary or functional aspects. Many books and overview articles have appeared for their description and characterization, many of which point to the importance to investigate patterns of this kind in the laboratory and by theoretical modeling and explanation [Winfree, 1987; Murray, 1993; Meinhard, 2009; Ortoleva, 1993; Davidenko et al., 1992; Lechleiter, Clapham, 1992; Graham et al., 1994; Dahlem et al., 2000].

A whole scientific field has thus developed concerned with structure formation in experimental model systems in the laboratory [Kapral, Showalter, 1995] and in conceptual approaches on the basis of nonlinear dynamics and non-equilibrium thermodynamics [Nicolis, Prigogine, 1977]. Here, we wish to focus on non-linear chemical reactions, in which self-organizing effects lead to temporal and spatial patterns with pronounced significance to biochemistry, biology, and biomedicine [Kapral, Showalter, 1995; Field, Burger, 1985].

These specific non-equilibrium systems have been frequently reviewed, and we will deal with the prototype of this type of systems, the Belousov-Zhabotinsky (BZ) reaction. This reaction, again, has been described in many contributions [Field, Burger, 1985; Tyson, 1976]. We restrict ourselves here to say that the behavior of this reaction is determined mostly by an activator and an inhibitor variable. The activator $\mathrm{H}_{2} \mathrm{BrO}_{2}$ undergoes an autocatalytic reaction. The inhibitor $\mathrm{Br}^{-}$, when present in an overcritical amount, will suppress the growth of the activator. Furthermore, the catalyst and redoxindicator ferroin participates in the reaction, being responsible for the color changes of the reaction between red (reduced state) and blue (oxidized state). The global behavior includes oscillations between red and blue in a stirred volume and wave propagation with blue fronts and red tails in thin layers extended in a Petri dish.

In fact, this way waves of excitation are generated, which are characterized by a resting unexcited (red) medium and an active (excited blue) travelling front followed by a period of refractoriness, quite analogous to pulses and waves in neuronal fibers and on the heart muscle. Obviously, there has been interest raised to use these chemical waves as a model for biological excitation processes, and this is a major reason to put the BZ-waves under experimental and theoretical scrutiny.

A major issue in current research, motivated by many applicative aspects, is the question, how to control the self-organized excitation waves by external means. Again, there are several overviews available, how to succeed in this intention, many of which deal with the influence of light impinging on systems with photosensitive catalysts and with changing the composition of the atmosphere [Steinbock, Müller, 1999]. In this work we will present earlier and recent results on the effect of externally applied electric fields, which have proven to be among the most efficient methods to this end.

Numerous chemical species in the complex BZ reaction mechanism are of ionic nature. The central species are the bulky iron complex ferroin, which carries a positive charge of two or three depending on its oxidation state, and the small negatively charged bromide ion. The autocatalytic species $\mathrm{HBrO}_{2}$ is another important actor taking part in the chemical events leading to wave propagation. This species however is electrically neutral. What happens to propagating waves and rotating spirals if an external electric field is applied?

Already in 1981 Feeney, Schmidt and Ortoleva performed experiments in which they applied parallel electric fields $(E \approx 10-50 \mathrm{~V} / \mathrm{cm})$ to spatially extended BZ media [Feeney et al., 1981]. They observed an increased velocity of waves propagating towards the positive anode, while waves propagating towards the cathode were decelerated. Ševčíková and Marek continued this work [Ševčíková, Marek, 1984], as well as other authors [Steinbock et al., 1992; Agladze, DeKepper, 1992]. It was found that at higher field strength waves can reverse their propagation direction or split in a fairly complex fashion [Ševč́ková et al., 1992, 1996], as we will see further below in the Result Section 3 (fig. 3). 
There exists evidence about the effect of an applied electric field in reactions other than the BZ system. Stationary Turing patterns in the so-called CIMA reaction have been subject to such fields and show a significant spatial drift [Schmidt et al., 2003]. Either well, comparable effects were observed in patterns of the PA-MBO reaction under the impact of electric currents [Münster, 2002].

Let us now focus on chemical patterns which are characterized by excitable dynamics, that is where traveling excitation waves occur. These waves have been subjected to an external electric field and the effects by that will be reported. There will be three parts of this kind of study: the first one referring to well established phenomena which have been observed a certain while ago, mainly in a twodimensional setting; a study of three-dimensional waves and influence on the filaments organizing the actual dynamics; finally, a presentation of most recent data on excitation waves, as they develop in microemulsion systems, and their response to the action of external electric fields.

Our overview will contain some remarks on the preparation procedure as well as sections summarizing the observed phenomena.

\section{Experimental}

\section{One- and two-dimensional systems}

For the work on systems in one or two dimensions there are several detailed descriptions of the employed experimental techniques [Steinbock et al., 1992; Ševčíková et al., 1992]. In all cases some standard BZ recipes were used, consisting of the chemical components sodium bromate, sodium bromide, malonic acid, sulfuric acid and ferroin as the catalyst and indicator. For one-dimensional investigations, the solution was placed in a rectangular cuvette and a field was applied via Pt plate electrodes immersed in appropriate side chambers. For details see [Ševčíková et al., 1992].

In the two-dimensional case a rectangular flat chamber containing a thin layer of the standard BZ solution was used with electrolyte-soaked paper strips serving as planar electrodes. The experimental setup is depicted in [Steinbock et al., 1992]. In all cases care was taken for an efficient temperature control to avoid effects from Ohmic heating. The modes of voltage- or current-control were compared to each other and undesirable electrode effects were avoided. For experimental details consult the cited references.

\section{Three-dimensional geometry}

For observations in three-dimensional setups we prepare the $\mathrm{BZ}$ solution with initial concentrations: $\left[\mathrm{H}_{2} \mathrm{SO}_{4}\right]=200 \mathrm{mM},[\mathrm{MA}]=50 \mathrm{mM},\left[\mathrm{NaBrO}_{3}\right]=50 \mathrm{mM}$, and [ferroin] $=0.625 \mathrm{mM}$. To prevent any hydrodynamic perturbation, the reaction is embedded in a $1.0 \% \mathrm{w} / \mathrm{w}$ agarose gel. In the absence of electrical forcing, thin layers of the BZ solution support rigidly rotating spiral waves. The experiments were performed using a rectangular reactor made from transparent plexiglas as shown in fig. 1 .

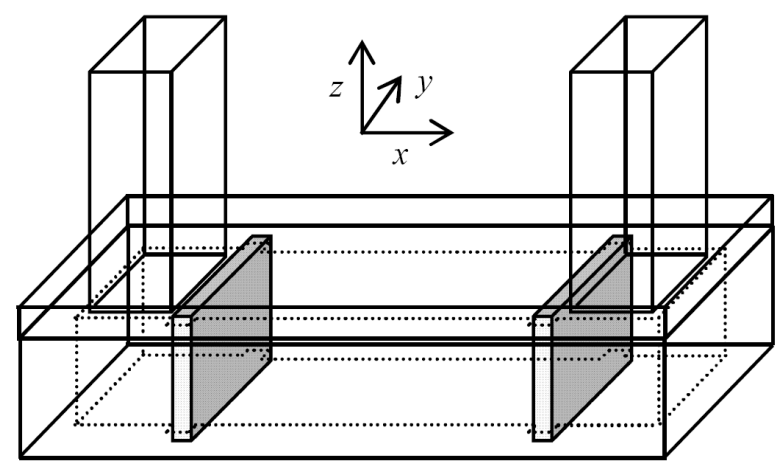

Fig. 1. Reactor for the study of the dynamics of scroll waves in an electrical current 
The BZ medium in a gel is placed in the $12 \times 12 \times 60 \mathrm{~mm}^{3}$ reaction volume into which a scroll ring is initiated. The longitudinal ends of the reactor chamber are connected to electrolytic compartments, which are filled with the BZ reaction of the same composition as that in the main compartment, except for the agarose (i.e., without a gel). The electrolytic compartments are separated from the main reaction chamber by porous glass filters to prevent any perturbation of the scroll ring by gas bubbles formed during electrolysis. The chimneys are utilized to release the bubbles. A constant electrical current is applied via two planar platinum plate electrodes $\left(10 \times 20 \mathrm{~mm}^{2}\right)$ along the $\mathrm{x}$ axis, i.e., pointing from one vertical planar electrode to the other. In most of our experiments, the strength of the current density $J$ is set to $40 \mathrm{~mA} \mathrm{~cm}{ }^{-2}$.

We use a two-layer strategy [Jahnke et al., 1988; Luengviriya et al., 2006] to create scroll rings with different orientation $\theta$ (the angle between the unit vector $S$ of the scroll ring and the vector of current density $J$ ). For the initial angle $\theta_{0}=90^{\circ}$, the reactor is set horizontally and a portion of the BZ solution is filled into as the first layer. Then a hemispherical wave front is initiated by immersion of an $\mathrm{Ag}$ wire of $0.25 \mathrm{~mm}$ diameter. The diameter of the hemispherical front grows with time and when it is about $2.5-3.5 \mathrm{~mm}$, a second layer of the medium is set on the top of the first layer. The circular edge of the hemispherical front curls in to form a scroll ring. The initial orientation $\theta_{0}$ can be adjusted by tilting the reactor with respect to the ground during the initiation.

We observe the dynamics of scroll rings from two perpendicular projections [Pertsov et al., 1993] of the wave structures using a single CCD camera. The reactor is mounted onto a support and placed into a plexiglass thermostating bath containing water with a constant temperature at $22 \pm 0.1{ }^{\circ} \mathrm{C}$. A mirror is placed with a tilt angle of $45^{\circ}$ underneath the reactor allowing a simultaneous observation of the lateral and vertical views of the reactor. A white light box is used to illuminate the BZ medium from the side. During the image acquisition, the video signal from the CCD camera (Hamamatsu, C3077) is digitized using a frame grabber card (Pulsar, Matrox). The resolution of the images is adjusted to $0.05 \mathrm{~mm} \mathrm{pixel}^{-1}$. The delay time between the stored images is $5 \mathrm{~s}$.

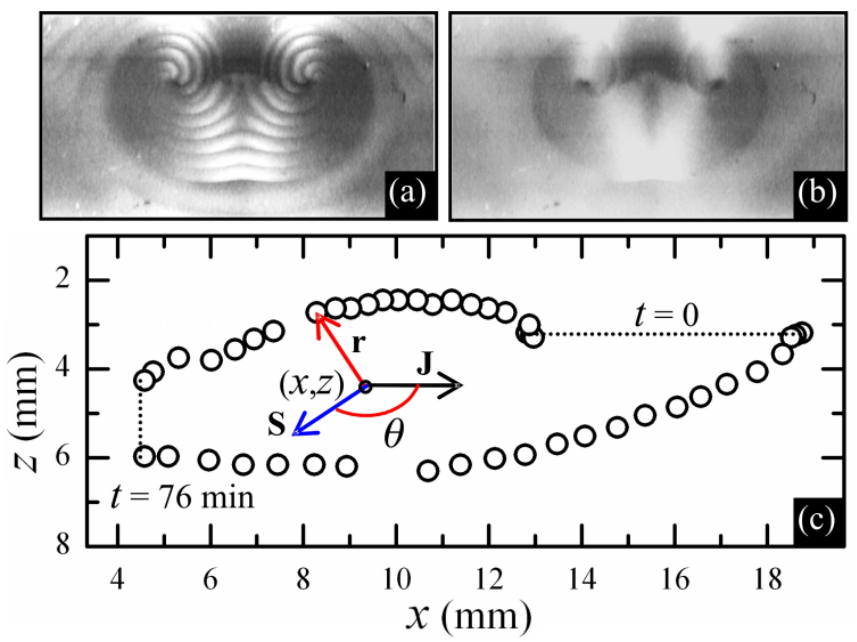

Fig. 2. Evaluation of the scroll ring dynamics in a BZ medium. ( $a$ and $b$ ) are superpositions of 7 and 40 images of the lateral projection of a scroll ring, respectively. Two small dark regions are the cores around which a pair of spiral waves rotates. (c) The scroll ring was subjected to an applied electrical current density. Circles show the positions of the core centers of two counter-rotating spirals in the lateral projection. The orientation $\theta$ is the angle between $S$ and $J$ (from [Luengviriya et al., 2008])

To evaluate the dynamics of scroll rings, we determine the cores of two counter-rotating spirals in the lateral projection by a superposition of an image series taken during one rotation. The cores are the dark areas never visited by the spiral waves. Figure $2 a$, which is a superposition of 7 images of the projection, shows a pair of counter-rotating spiral waves rotating around the cores. The wave fronts disappear when 40 images are superposed leaving only the cores as shown in Figure $2 b$. The core centers of the two counter-rotating spiral waves are estimated by eye and the straight line connecting them 
is taken as the diameter of the scroll ring filament as shown in figure $2 c$. This allows the determination of the radius $r$, and the center $(x ; z)$ of the scroll ring. The unit vector $S$ is perpendicular to the radius $r$ (and accounts for the direction of spiral rotation) and finally the orientation $\theta$ can be measured.

\section{Microemulsions}

Experiments are performed by using the ferroin- or the bathoferroin-catalyzed BZ reaction dissolved in an aerosol OT (AOT; sodium bis(2-ethylhexyl)sulfosuccinate) water in-oil microemulsion with octane as solvent (from Sigma-Aldrich).

Two different microemulsions are prepared, having the same $\omega\left(\omega=\left[\mathrm{H}_{2} \mathrm{O}\right] /[\mathrm{AOT}]\right.$, where $\left[\mathrm{H}_{2} \mathrm{O}\right]$ and [AOT] are the molar concentrations of $\mathrm{H}_{2} \mathrm{O}$ and AOT, respectively) and the droplet fraction $\varphi_{d}$ $\left(\varphi_{d}=\left(\mathrm{VAOT}+\mathrm{VH}_{2} \mathrm{O}\right) / V_{\text {total }}\right.$, where VAOT, $\mathrm{VH}_{2} \mathrm{O}$, and $V_{\text {total }}$ are the volumes of AOT, $\mathrm{H}_{2} \mathrm{O}$, and the total ME, respectively) [Schwartz et al., 1999]. They are mixed with a ratio of 1:1 and a certain volume of oil, depending on the requested droplet fraction. The used recipes are listed in table 1.

A small amount of this reactive microemulsion is sandwiched between two plexiglass plates with a platinum-iridium foil $(90 / 10 \mathrm{wt} \%)$, having a thickness of $100 \mu \mathrm{m}$ or 220 , at two opposite sides of the glass plates. This foil serves as spacer between the plates and as electrodes (length $2.5 \mathrm{~cm}$ for $100 \mu \mathrm{m}$ thickness and $3.0 \mathrm{~cm}$ for $220 \mu \mathrm{m}$ thickness) for the application of an electric field. The platinum-iridium foil is chosen, since platinum is chemically inert and no electrolysis could be observed during the experiments.

The electrodes are placed at a distance of 3.0 and $4.5 \mathrm{~cm}$ parallel to each other, such that an almost homogeneous field could be assumed. A voltage between $31.2 \mathrm{~V}$ and $39.2 \mathrm{~V}$ is applied, leading to an electric field between $8.7 \mathrm{~V} \mathrm{~cm}^{-1}$ and $13 \mathrm{~V} \mathrm{~cm}^{-1}$. The current lies between 0.3 and $1.5 \mu \mathrm{A}$.

The patterns developing in the reactor are illuminated by a LED panel through a $488 \mathrm{~nm}$ interference filter and observed with a microscope equipped with a digital CCD camera (AVT Stingray) connected to a computer. All experiments are carried out at a temperature $21 \pm 1{ }^{\circ} \mathrm{C}$.

Table 1. Concentrations of the reactants in the ferroin-catalyzed BZ-AOT system in octane with ferroin and BP as catalysts for different $\omega$ and $\varphi_{d}$

\begin{tabular}{cccccccc}
\hline catalyst & $\begin{array}{c}\text { Layer } \\
\text { thickness } \\
{[\mu \mathrm{m}]}\end{array}$ & $\begin{array}{c}\mathrm{MA} \\
{[\mathrm{M}]}\end{array}$ & $\begin{array}{c}\mathrm{H}_{2} \mathrm{SO}_{4} \\
{[\mathrm{M}]}\end{array}$ & $\begin{array}{c}\mathrm{NaBrO}_{3} \\
{[\mathrm{M}]}\end{array}$ & $\begin{array}{c}\text { Ferroin/ } \\
\mathrm{BP}[\mathrm{mM}]\end{array}$ & $\omega$ & $\varphi_{\mathrm{d}}$ \\
\hline Bathoferroin & 220 & 0.291 & 0.195 & 0.175 & 4.94 & 14.9 & 0.7 \\
Bathoferroin & 100 & 0.242 & 0.194 & 0.174 & 6 & 12 & 0.455 \\
Ferroin & 100 & 0.207 & 0.259 & 0.155 & 1.74 & 18.03 & 0.43 \\
\hline
\end{tabular}

\section{Results}

\section{One-dimensional waves}

The most remarkable finding in the one-dimensional case of electric field effects is that of reversal of propagation direction [Ševčíková, Marek, 1984; Ševčíková et al., 1992]. Normally, a travelling excitation wave would keep its direction, because the refractory zone in its wake would not allow any backward motion. With the action of an electric field, however, this becomes possible, as shown in figure $3 a$. The velocity of a propagating front is reduced when a field is applied with a polarity counteracting the overall motion of the negatively charged inhibitor bromide ion. If the strength of the field is sufficiently high, it can reverse the bromide ion drift direction, causing the front also to move backwards with respect to its original propagation direction.

Beyond that, it may also occur that at the point of reversal the wave splits into two parts: one of them continuing along the original path, the other reversing its direction (fig. $3 b$ ). Both observations, 
the reversal and the splitting of an excitation wave demonstrate the high potential of electric fields to influence and control wave dynamics that normally proceeds along its own, self-organized path. The usual scenario of excitation kinetics is changed by external forcing such that at the reversal point a transient bistable situation prevails, which offers the possibility of choosing between two options for further travel direction. This change of the local dynamics of the system is evoked by the interaction of the electric field of the proper intensity and polarity with the concentration gradients along the traveling wave.

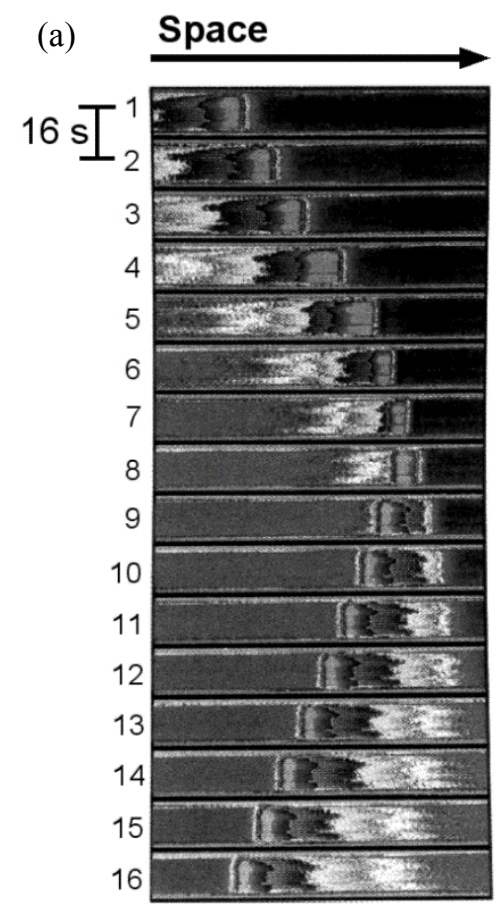

(b)

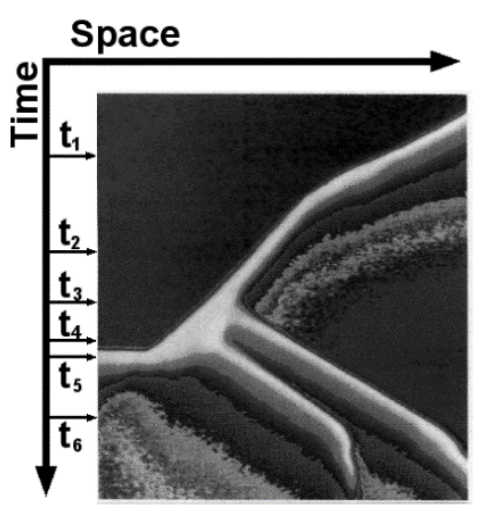

Fig. 3. (a) Reversal of a chemical front in the BZ-reaction under the action of an electric field. The wave travels in a rectangular cuvette and is observed from the side. The time series of video images is taken at 16-s intervals. The polarity was changed after time step 3 such that the electric field is now oriented from the cathode to the right to the anode to the left. It is such that the negative electrode is located in front of the initial direction of wave propagation, thus exerting a repulsive force against the negatively charged bromide ion in the front. (b) Time-space plot of the splitting of a front. Experimental conditions as in (a). With an electric field of appropriate strength and polarity, a wave front may continue along its original direction and, at the same time, follow one or more reversed paths (from [Ševčíková et al., 1992])

\section{Two-dimensional waves}

We now turn to excitation waves evolving in thin layers (typical thickness in the mm range) extended in a flat dish, where a more complex dynamical behavior is found. Basically one finds target patterns and rotating spirals, but scenarios of wave turbulence and irregular decomposition patterns are also common [Kapral and K. Showalter, 1995; Steinbock et al., 1992]. In particular, we have investigated pairs of spiral waves, which under free conditions rotate with opposite sense of rotation, each around a spatially fixed core region.

Figure 4 illustrates the effect of an externally applied electric field on such a pair of spiral waves in the ferroin-catalyzed BZ reaction embedded in agar gel [Steinbock et al., 1992]. In these experiments a constant electric filed $(E=0-6 \mathrm{~V} / \mathrm{cm})$ was applied to the $\mathrm{BZ}$ gel system via two parallel electrodes, which were realized as simple salt bridges to avoid the contamination of the BZ medium by products of undesired electrochemical reactions. A typical snapshot of a pair of such drifting waves (anode oriented parallel to the bottom side of the figure) is shown in figure $4 a$. The central finding is that the spiral waves are drifting towards the anode [Steinbock et al., 1992]. 
The overall drift towards the anode has an additional component perpendicular to the field resulting in trajectories as depicted in figure $4 b$. The direction of this perpendicular drift depends on the chirality of the spirals. Although the trajectories of both spirals point in $-x$ direction, one finds that the clockwise rotating pattern is also pulled to the left, while the counterclockwise spinning wave is pulled to the right. Changing the polarity of the field causes a drift back towards the initial locations. Note that the drift shown in graph $B$ does not exactly occur along a straight line, but is rather characterized by a continuous trajectory with successive loops.

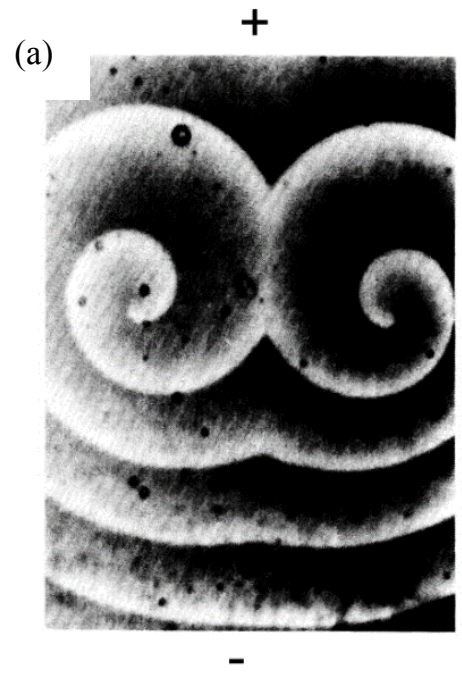

(b)

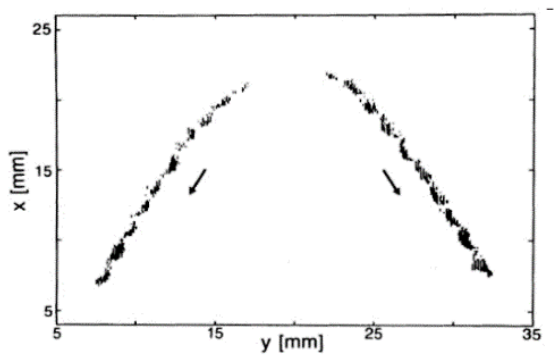

Fig. 4. A pair of spiral waves in the ferroin-catalyzed BZ system is perturbed by a constant electric field. Field lines are parallel and oriented vertically with the anode located at the bottom side of the figures. The electric field induces a spiral drift towards the anode and a strong deformation of the Archimedian spiral geometry. (a) Snapshot of the pair of drifting spirals. (b) Trajectory of the corresponding spiral tips (from [Steinbock et al., 1992])

Another interesting phenomenon that has been observed in experiments on electric-field induced spiral drift is the deformation of the spiral geometry [Steinbock et al., 1992]. While spiral waves in unperturbed systems have usually an Archimedian shape (i.e., constant pitch), here the drift of the tips is generating variations of the wavelength. The deviations from the unperturbed wavelength reach a maximum in the back of the drift direction (compare fig. 4a). Apparently, the different wavelengths in the upper and lower part of this image are due to a Doppler effect.

We note that the perturbation of chemical wave patterns by electric fields can be efficiently used for an external control of wave propagation. An interesting example for exploiting spiral drift is discussed in the following paragraph: Since it is possible to induce an outward oriented spiral drift (see fig. $4 b$ ), one should also be able to send a pair of spirals on collision course.

We see in figure 5 a sequence of four snapshots illustrating the outcome of such a spiral wave collision [Schütze et al., 1992]. Snapshot $(a)$ shows the initial wave pattern consisting of a pair of counter-rotating spirals that have nearly identical size and phase. A constant electric field is applied to the BZ gel system with the anode located parallel to the right side of the subfigure. The field is now pulling the spirals towards the anode and is decreasing the relative distance between the spiral tips (figure $5 b$ ). At a certain critical distance («wavelength) the spiral tips annihilate, thus removing the spinning pacemakers from the system $(c)$.

The resulting unexcited area in the former region of tip rotation triggers a new pattern of low frequency (image $d$ ), since the bulk dynamics in this particular BZ system is, in fact, not excitable but oscillatory, with rather long oscillation period. These intrinsic oscillations of the BZ bulk had been earlier suppressed by the high-frequency spiral waves. Now, where the spirals have vanished, the system creates an autonomous pacemaker according to the local phase gradients created by the last spiral 
rotation. The collision of a spiral pair has therefore led to spiral annihilation and eventually to the creation of a target pattern with its pacemaker located in the spiral collision region.
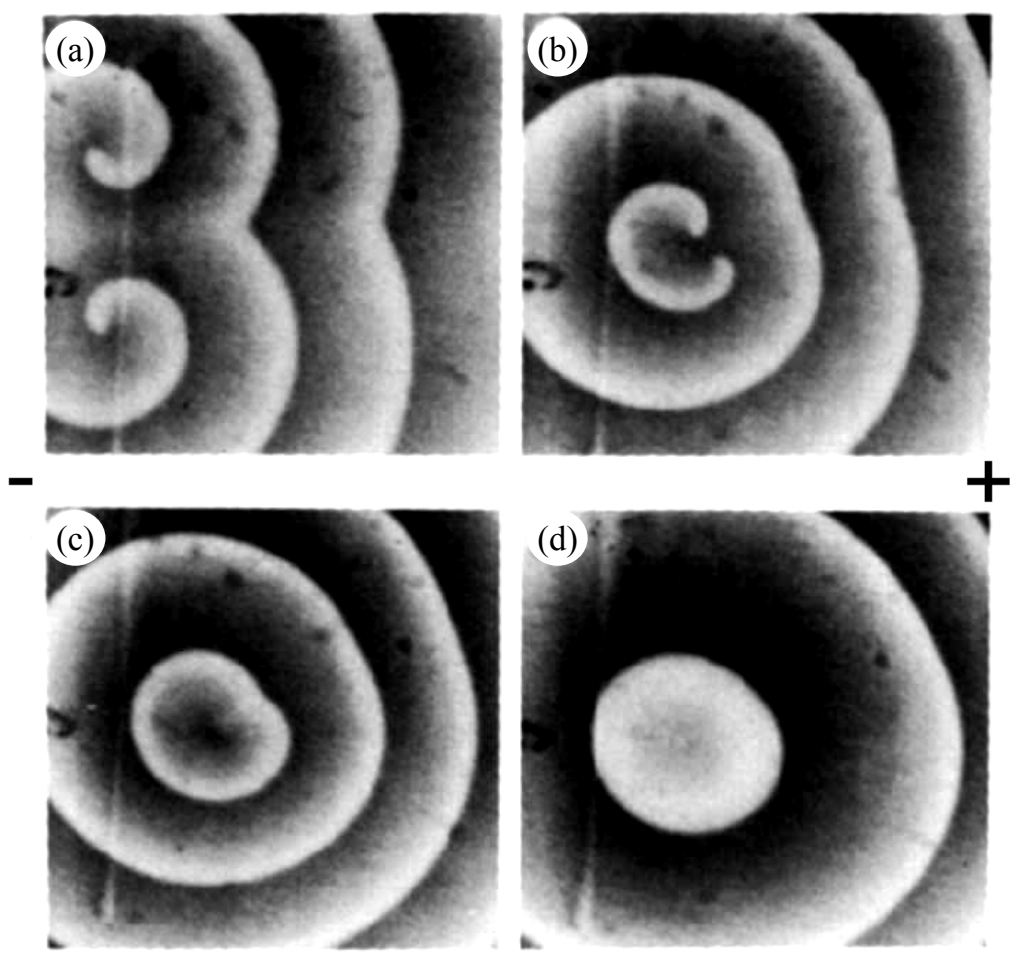

Fig. 5. Spiral wave annihilation due to an electric-field induced drift. The electric field is oriented from the anode to the right to the cathode to the left. Opposite perpendicular motions of spiral tips in the initial structure $(a)$ reduce their relative distance $(b)$. Annihilation occurs, when the separation distance of the tips is below a certain critical size $(c)$. Afterwards one observes the birth of a non-rotation trigger wave $(d)$ in the central region of spiral wave annihilation. The location of its pacemaker is determined by the local phase information created by the last spiral rotation (from [Schütze et al., 1992])

\section{Three-dimensional experiments}

We now review experiments on the dynamics of scroll rings in three-dimensional BZ media in the presence of an applied electrical current [Luengviriya et al., 2006; Luengviriya, Hauser, 2008]. Under these conditions, the scroll rings are subjected to two simultaneous processes, namely an intrinsic contraction and a possible reorientation with respect to the direction of the applied current. Due to the intrinsic contraction, scroll rings may self-annihilate before they reach a stationary alignment. To investigate the dynamics for the entire range of orientations, experiments with various initial orientations were conducted such that the intervals of the initial and final orientations from different experiments overlapped.

We now consider an example of a scroll ring as observed from two perpendicular projections, the top and lateral views, shown in figure $6 a-c$. The scroll ring is subjected to an electrical current density of $J=40 \mathrm{~mA} \mathrm{~cm}$. The direction of the applied current pointed to the right of the figure. The filament initially lied in a horizontal plane and its unit vector S points downwards forming a right angle with the current density vector J (fig. $6 a$ ). Hence, the initial scroll ring appears as circular wave fronts and two counter-rotating spirals in the top and lateral projections, respectively. Due to the effect of the applied current, the filament plane rotates (fig. $6 b$ and c). During the reorientation, the shape of wave front near the filament of the scroll ring in the top projection changes from a circle to an ellipse (fig. 6b) and finally to two counter-rotating spirals (fig. $6 c$ ) while the scroll ring in the lateral view appears as two counter-rotating spirals for the whole experiment. 


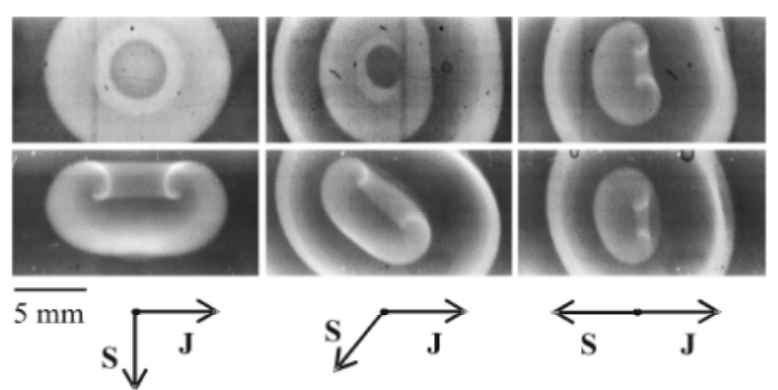

(a) 0 min

(b) $37 \mathrm{~min}$

(c) $70 \mathrm{~min}$

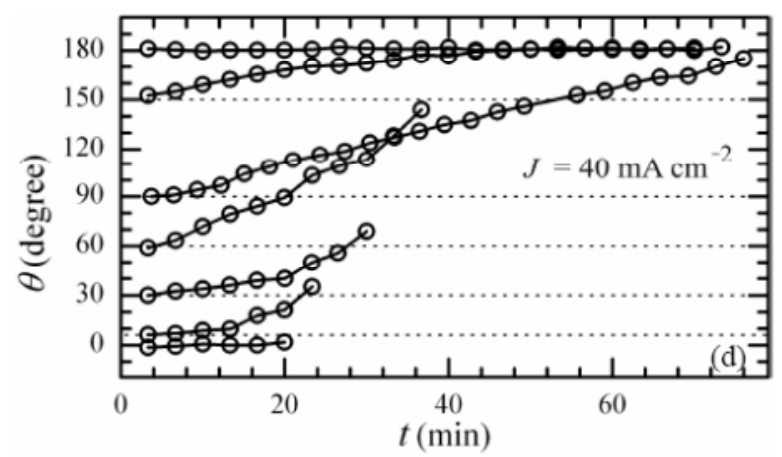

Fig. 6. Reorientation of a scroll ring under an applied electrical current in the BZ reaction. $(a-c)$ The filament reoriented from $90^{\circ}$ to $180^{\circ}$ (from [Luengviriya et al., 2006]). (d) Evolution of different orientation $(\theta)$ in the course of time (from [Luengviriya, Hauser, 2008])

The reorientation in figure $6 a-c$ is accompanied by a linear drift towards the positive electrode (to the left of the figure) with a rate of $0.17 \mathrm{~mm} \mathrm{~min}^{-1}$, and a slight downward drift (rate = $=0.03 \mathrm{~mm} \mathrm{~min}^{-1}$ ). At the end of the experiment, $S$ is anti-parallel to $J$ before the scroll ring disappears due to contraction and subsequent self-annihilation. A deformation of the uniform scroll ring due to the reorientation can be observed from the lateral projection. During the whole experiment, the scroll ring appears as a pair of spirals in this lateral projection, however, the shape of spirals becomes asymmetrical. Such a behavior has been observed earlier in experiments using a thin layer of the BZ reaction [Steinbock et al., 1992].

To study the influence of the electrical current on the scroll rings at other orientations, experiments were performed using different initial angles $\theta_{0}$. The initial radii $r_{0}$ of the scroll rings were about $2.6-3.3 \mathrm{~mm}$. Figure $6 d$ shows the scroll ring orientation in the experiments with initial angles $\theta_{0}=0^{\circ}$, $6^{\circ}, 30^{\circ}, 60^{\circ}, 90^{\circ}, 150^{\circ}$, and $180^{\circ}$. In many of these experiments, the scroll rings contracts and selfannihilates while the reorientation is still in progress. By varying $\theta_{0}$, we have investigated the dynamics of the scroll rings in overlapping ranges of orientation. For $6^{\circ}<\theta_{0}<180^{\circ}$, the scroll rings are found to change their orientation. In fact, the angle increases towards $180^{\circ}$. When $\theta_{0}=0^{\circ}$ or $\theta_{0}=180^{\circ}$, the orientation of the scroll rings remains unchanged. These results indicate that both parallel and antiparallel orientations $\left(\theta_{0}=0^{\circ}\right.$ or $\left.\theta_{0}=180^{\circ}\right)$ are stationary states. As every angle $\theta_{0}>6^{\circ}$ leads to an increase of the angle towards $180^{\circ}$, the anti-parallel orientation of the scroll ring unit vector $S$ with respect to the current $J$ is a stable solution of the system. By contrast, the parallel orientation is also a stationary solution, however, it represents an unstable stationary state, since small derivations will induce a reorientation of the scroll ring.

At the end of this section, we show that the scroll rings may be controlled efficiently in their size by using the electrical current. This relies on the fact that the strength and direction of the applied current can be changed within a short time. Figure 7 shows the evolution of a scroll ring under a stepwise variation of the electrical current at an anti-parallel orientation $\left(\theta_{0}=180^{\circ}\right)$. The plots show the positions of the scroll ring (i.e., the core centers of counter-rotating spiral pair at $200 \mathrm{~s}$ intervals) which 
drifts to the right of the figure while $J$ points to the left. Starting with $J=40 \mathrm{~mA} \mathrm{~cm}{ }^{-2}$, the scroll ring

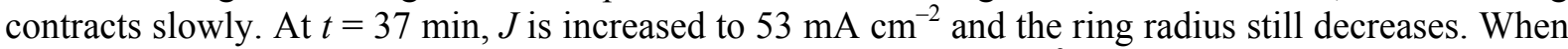
the radius is about $1 \mathrm{~mm}, J$ is increased drastically to $106 \mathrm{~mA} \mathrm{~cm}{ }^{-2}$. This strong forcing leads to an expansion of the scroll ring. Note that under a weaker field, e.g., $J=40 \mathrm{~mA} \mathrm{~cm}{ }^{-2}$, the scroll ring with radius about $1 \mathrm{~mm}$ would self-annihilate in a short time. Finally, the current is switched off at $t=67 \mathrm{~min}$, thus causing the scroll ring to shrink again.

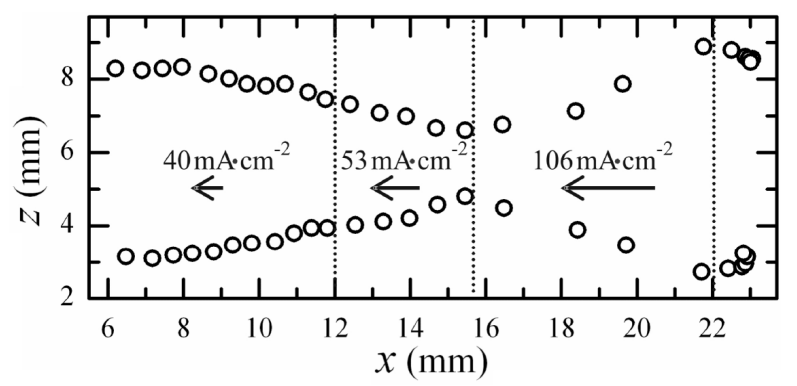

Fig. 7. Manipulation of a scroll ring by a stepwise variation of the electrical current. Circles show the position of core centers of two counter-rotating spirals in the lateral projection at $200 \mathrm{~s}$ intervals (from [Luengviriya at al., 2008])

\section{Microemulsions}

When the BZ-reaction is embedded in a microemulsion (ME), in particular with the catalyst bathoferroin (BP), a rich variety of patterns can be found, which is surpassing by far the complexity found in the standard preparation in water or in an aqueous gel, as considered in the previous sections. One finds so-called dash waves and discontinuously propagating waves (jumping, rotating and bubble waves). The difference concerning the patterns found in the ferroin-catalyzed BZ-AOT system might occur due to the more hydrophobic character of the BP, which is not soluble in water. Its molecules are located mostly in the hydrophobic AOT shell, yielding a clustering of nanodroplets [Dähmlow et al., 2014].

By applying an electrical field across the spatially extended solution layer, a linear drift of the patterns can be observed, depending on the field strength and the layer thickness of the ME.

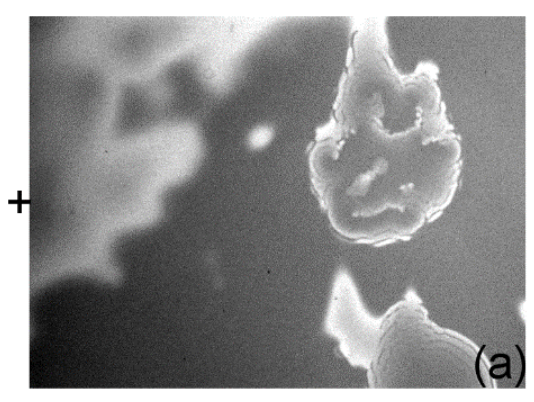

$\mathrm{t}=321.0 \mathrm{~min}$

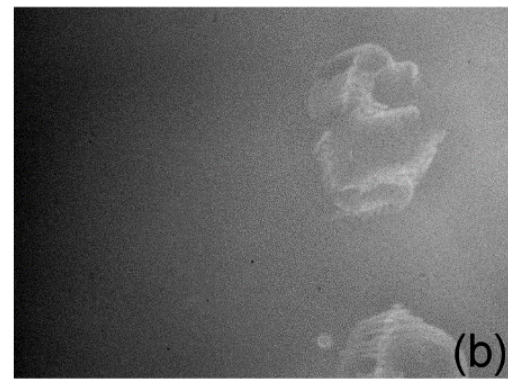

$\mathrm{t}=337.5 \mathrm{~min}$

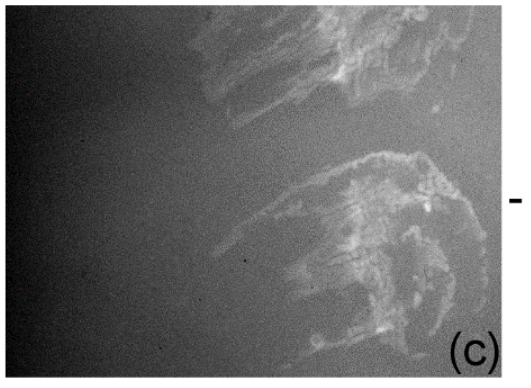

$\mathrm{t}=363.2 \mathrm{~min}$

Fig. 8. Smearing of patterns in the bathoferroin-catalyzed BZ-AOT system at different instants in time and a layer thickness of $220 \mu \mathrm{m}$. (a) Jumping and rotating waves with coexisting bulk oscillations in the system before an electric field was applied. $\left(b\right.$ and $c$ ) Immediately after the application of the electric field $\left(10 \mathrm{~V} \mathrm{~cm}^{-1}\right)$ the patterns start to drift diagonal to the field lines towards both electrodes. Concentrations: $[\mathrm{MA}]=0.291 \mathrm{M}$, $[\mathrm{NaBrO} 3]=0.175 \mathrm{M},\left[\mathrm{H}_{2} \mathrm{SO}_{4}\right]=0.195 \mathrm{M}$, and $[\mathrm{BP}]=4.94 \mathrm{mM}, \varphi_{d}=0.7, \omega=14.9$; image size: $11.7 \times 8.6 \mathrm{~mm}^{2}$

In figure 8 the layer thickness is $220 \mu \mathrm{m}$. Rotating and jumping waves can be observed together with coexisting bulk oscillations as shown in figure $8 a$. At the bottom of the image, the jumping waves developed from a target pattern and the upper pattern from some pairs of counter rotating spiral waves. After applying an electrical field, the patterns show a drift into both directions: towards the positive 
and the negative electrode, with a certain angle with respect to the field lines (fig. $8 b$ and $8 c$ ). The upper pattern shows a larger inclination to the field lines, than the lower one, since the rotation of the spirals governs the drift [Steinbock et al., 1992]. The patterns drift into two directions (they are "smearing out") as a result of the layer thickness $(220 \mu \mathrm{m})$ of the ME. The sodium ion of the surfactant AOT, which is located inside the water droplet, is positively charged and can polarize the droplets [Eicke, Naudts, 1987], such that a drift towards the negative electrode occurs (right side of fig. 8), whereas the inhibitor is negatively charged, which leads to a drift towards the positive electrode.

In the time-space plot of the described patterns, the vertical drift into both directions is clearly observable (fig. 9). The vertical bright lines are the bulk oscillations (BO) in the system and the lower broken lines correspond to jumping waves. These clear wave structures are instantaneously destroyed after the application of the electric field.

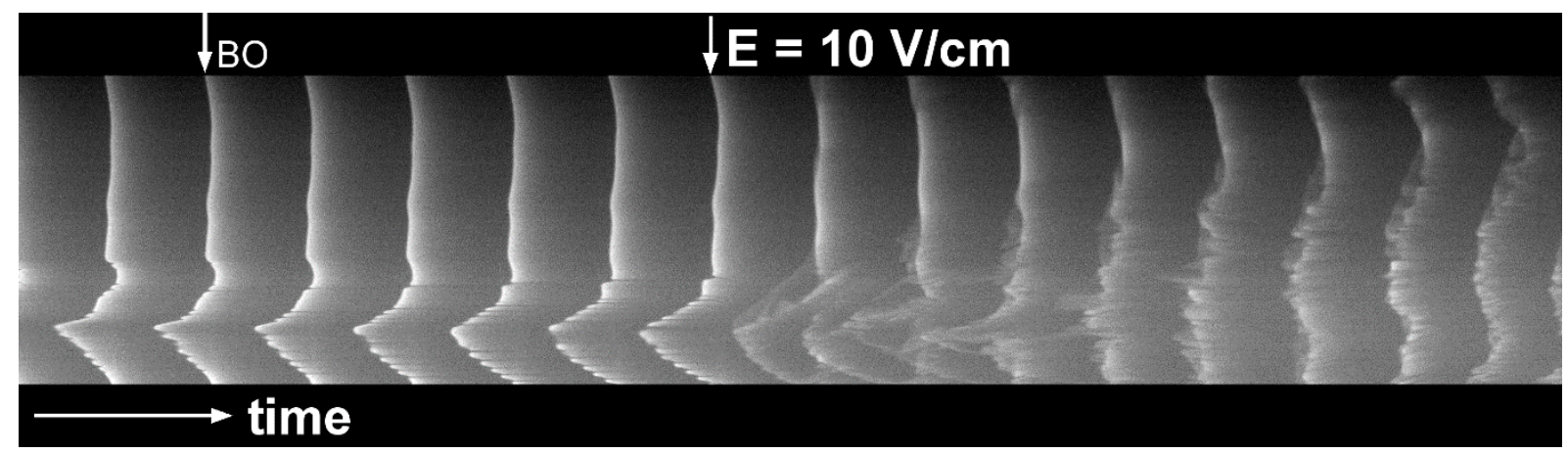

Fig. 9. Time-space plot from 140.51 to 237.18 min of fig. 8 along a horizontal line through the upper part of the pattern. The bulk oscillations (BO) are the vertical lines and the broken lines correspond to jumping waves. After the field application $\left(10 \mathrm{~V} \mathrm{~cm}^{-1}\right)$, the patterns drifted towards the positive and negative electrode

A layer thickness of $100 \mu \mathrm{m}$ leads to a clearly observable drift of patterns towards the positive electrode. In figure 10, some pairs of counter-rotating spiral pairs are shown. The upper pair drifted towards the positive electrode, directly after the application of the field. Due to the interaction with neighboring spirals, the upper spiral pair moved towards the lower left corner of the recorded image, where the drift velocity increased significantly (fig. 10c). According to the layer thickness of $100 \mu \mathrm{m}$, no "smearing" of patterns is detected.
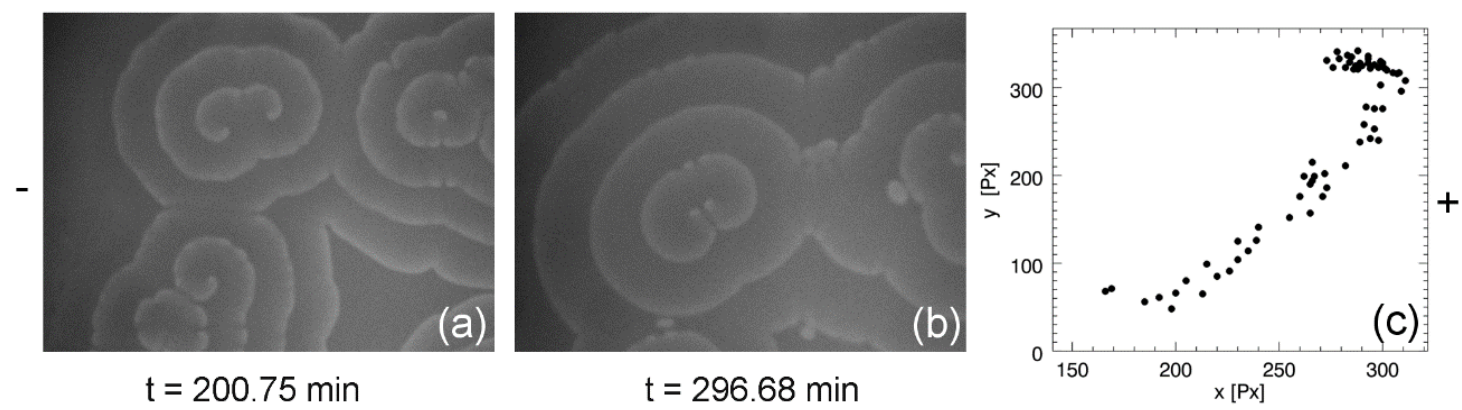

Fig. 10. $(a$ and $b$ ) Drift of patterns in the bathoferroin-catalyzed BZ-AOT system with a layer thickness of $100 \mu \mathrm{m}$. (c) The tracked trajectory of the upper spiral wave after the application of the electric field $\left(8.7 \mathrm{~V} \mathrm{~cm}^{-1}\right)$. Concentrations: $[\mathrm{MA}]=0.242 \mathrm{M},[\mathrm{NaBrO} 3]=0.174 \mathrm{M},\left[\mathrm{H} 2 \mathrm{SO}_{4}\right]=0.194 \mathrm{M}$, and $[\mathrm{BP}]=6 \mathrm{mM}, \varphi_{d}=0.455$, $\omega=12$; image size: $5.4 \times 4.1 \mathrm{~mm}^{2}$.

\section{Discussion}

The action of an electric field on pattern-forming chemical and biological systems belongs to the most efficient tools to influence and to control the inherent, self-organized dynamics of such com- 
plex "organisms". This is mostly due to the fact that there one finds many electrically charged components which govern the spatio-temporal organization. Thus, dominating ingredients like activating and inhibiting species are subjected to the impact of external forcing, as long as they carry an electric charge. This is true for the case of the BZ-reaction, where the bromide ion assumes the important role of inhibitor that rules, due to its negative charge, the resulting forced dynamics to a large extent. This predominant role is corroborated by the numerical modeling of a realistic scheme for the BZ reaction, the Oregonator model [Kapral, Showalter, 1995; Tyson, 1976]. In other nonlinear chemical reactions we find, as well, important charged compounds that readily respond to the action of external fields. This leads, for instance, to a spatial drift of otherwise stationary Turing patterns [Schmidt et al., 2003; Münster, 2002].

Our findings in the investigation of the BZ reaction under external electrical forcing comprise a number of remarkable phenomena: reversal of wave propagation, wave splitting, change of propagation velocity, drift of spiral waves with Doppler effect, annihilation of counter-rotating spiral waves, creation of new pacemaker centers, reorientation of scroll filaments, control of scroll ring size, drift and smearing out of various Turing patterns, as well as discontinuously propagating and dash waves evolving in microemulsions. So far, we do not know of any other method that can claim to produce a higher diversity of such pronounced effects in the systems under consideration.

Many authors have noticed, how important the application of such a tool should be for other excitable media, especially biological ones, where a disturbance of the excitable kinetics may lead to functional disorder and disease. For the healthy and constructive cases, we mention the life cycle of social amoebae in which the front dynamics determines to a large extent the survival strategy of this "simple" organism. To exert too much external stress on it though, may destroy major steps of this strategy and cause an interruption of the cycle. The field of morphogenesis, in general, offers many examples, too [Meinhard, 2009; Kapral, Showalter, 1995].

If we proceed to organs or organisms of much higher complexity, we may consider the heart muscle, where rotating activity of muscle excitation can lead to dangerous heart arrhythmia. Here one would wish to have a well controllable tool to influence such unhealthy excitation patterns in a smooth and efficient way. Ideally, one could think of a spiral annihilation effect as demonstrated in figure $3 b$. Its clinical realization, however, is far from realistic at the time. Finally, our brain is a huge organism containing excitable nerve fibers that interact with millions of other ones via excitatory and inhibitory synaptic connections. It is likely that this interaction is disturbed in diseases like migraine and epilepsy through disequilibrium of ionic species, among other sources of dysfunction.

In the case of embedding the BZ reaction in a microemulsion one can control the diffusion process of activator and inhibitor variable in a way that Turing-type patterns emerge (just choose the diffusion velocity of the inhibitor to be larger than that of the activator). Since Turing patterns are well known to occur in morphogenesis, i.e. on animal skins or in insect eggs [Kondo, Miura, 2010], microemulsions appear as suitable model systems to investigate perturbations from the outside such as gradients formed by an electric field. The application of electric field to the ferroin-catalyzed BZ-AOT system has been rarely investigated. In the past, structural changes in water-in-oil microemulsions with the ionic surfactant AOT were studied, which changes reversibly with the variation of physical parameters [Dähmlow et al., 2014]. Clusters of water droplets show an elongated shape in the direction of the electric field [Bedford, Ilgenfritz, 2000]. The increase in temperature and the field application changes the percolation of the microemulsion and a temperature induced percolation curve is shifted to higher temperatures in the electric field [Schlicht et al., 1996].

In the BZ-AOT system, below the percolation transition, an electric field induces a drift of Turing structures or of other wave patterns towards the positive electrode [Carballido-Landeira et al., 2012]. A field of $10 \mathrm{Vcm}^{-1}$ is applied in this case, such that the system is below the percolation. The wavelengths of the structures show minor changes by the influence of the applied field strength [Carballido-Landeira et al., 2012].

Finally, if one considers the neurons in the brain as a huge and complex network of interacting neurons and thus as a particularly large and potent excitable medium, one can ask the question about the role of electric fields. The idea has been formulated that these field are of major importance when 
it comes to understand long-range interactions between neurons. Microemulsions and their percolation properties under the influence of an electric field are considered as model systems for better understanding these long-range effects [Dähmlow et al., 2014].

The microscopic driving force of the observed phenomena under field action is electromigration of ionic species. The underlying electric force can result in a local change of the concentration $\mathrm{c}$ that is given by the equation

$$
\partial c / \partial t=\mu E \nabla c
$$

where $E$ is the electric field vector and $\nabla \mathrm{c}$ the gradient vector of the local ion concentration. The parameter $\mu$ is the ionic mobility of the charged species, defined as the proportionality factor between the velocity of ions and the applied electric field. Hence, the value of $\mu E$ corresponds to the migration velocity of the particular ion in an electric field $E$. By adding the right hand term of equation above to the set of reaction-diffusion equations describing the chemical system (as done many times in analytical and numerical work on excitation waves), one obtains a mathematical model that is suitable for the numerical simulation of experimental data. On this basis, numerous numerical studies have been carried out to reproduce and understand the effects of electric fields on pattern formation in chemical systems. Generally, these investigations are in good agreement with experiments, although certain quantitative features of numerical studies are not quite satisfactory yet. For instance, it may appear as a problem that in the two-variable version based on the Oregonator model, one usually takes the autocatalytic species $\mathrm{HBrO}$ as the excitation (active) variable and the catalyst ferroin as the recovery (inhibitory) variable. It might seem unreasonable to use an uncharged component to describe electrically induced flux. But in fact, because of the reaction kinetics, the dynamical behavior of $\mathrm{Br}$ - is closely related to that of $\mathrm{HBrO}_{2}$ [Field Burger, 1985; Schütze et al., 1992].

As in the last few decades, investigating the impact of external perturbations on the selforganized dynamics of reactive species has remained a relevant activity in nonlinear science. One can look forward to discovering still many remarkable phenomena in this field and witness their applicability in the natural sciences, engineering and biomedicine.

\section{References}

Agladze K. I., DeKepper P. Influence of electric field on rotating spiral waves in the BelousovZhabotinskii reaction, J. Phys. Chem. 96, 5239 (1992).

Bedford O. D., Ilgenfritz G. Electric field effects in AOT w/o microemulsions: Field-induced percolation and dynamics of structure changes, Colloid Polym. Sci. 278, 692-696 (2000).

Carballido-Landeira J., Taboada P., and Muñuzuri A. P. Effect of electric field on Turing patterns in a microemulsion, Soft Matter 8, 2945-2949 (2012).

Dahlem M. A., Engelmann R., Löwel S., Müller S. C. Does the migraine aura reflect cortical organization?, Eur. J. Neurobiology 12, 767-770 (2000).

Dähmlow P., Vanag V. K., Müller S. C. Effect of solvents on the pattern formation in a BelousovZhabotinsky reaction embedded into a microemulsion, Phys. Rev. E 89, 010902(R) (2014)

Davidenko J. M., Pertsov A. V., Salomonsz R., Baxter W., Jalife J. Stationary and drifting spiral waves of excitation in isolated cardiac muscle, Nature 355, 349-351 (1992).

Eicke H. F., Naudts $J$. Non-Linear Field effects due to activation-energy-controlled charge transport in microemulsions, Chem. Phys. Lett. 142, 1 (1987).

Feeney R., Schmidt S., Ortoleva P. Experiments on electric field-BZ chemical wave interaction: Annihilation and the crescent wave, Physica D 2, 536-544 (1981).

Field R. J., Burger M. (eds.) Oscillations and traveling waves in chemical systems, Wiley, New York (1985).

Graham M. D., Kevrekidis I. G., Asakura K., Lauterbach J., Krischer K., Rotermund H.-H., Ertl G. Effects of boundaries on pattern formation: Catalytic oxidation of $\mathrm{CO}$ on platinum, Science 264, 80-82 (1994). 
Jahnke W., Henze C., Winfree A. T. Chemical vortex dynamics in three-dimensional excitable media. Nature, 336, 662 (1988).

Kapral R., Showalter K. (eds.) Chemical waves and patterns, Kluwer, Academic Press, Dordrecht (1995).

Kondo S., Miura T. Reaction-Diffusion Model as a Framework for Understanding Biological Pattern Formation, Science 329, 1616 (2010).

Lechleiter J. D., Clapham D. E. Molecular mechanisms of intracellular Calcium excitability in X. leavis oocytes, Cell 69, 283-294 (1992).

Luengviriya C., Hauser M. J. B. Stability of scroll ring orientation in an advective field. Phys. Rev. E 77, 056214 (2008).

Luengviriya C., Müller S. C., Hauser M. J. B. Reorientation of scroll rings in an advective field, Phys. Rev. E, 77, 015201 (2008).

Luengviriya C., Storb U., Hauser M. J. B., Müller S. C. An elegant method to study an isolated spiral wave in a thin layer of a batch Belousov-Zhabotinsky reaction under oxygen-free conditions. Phys. Chem. Chem. Phys. 8, 1425 (2006).

Meinhard $H$. The algorithmic beauty of seashells. Springer, Berlin (2009).

Münster A. Simulation of stationary chemical patterns and waves in ionic reactions, Discrete Continuous Dynamical Syst. Ser. B 2, 1 (2002).

Murray J. D. Mathematical Biology, Springer, Berlin (1993).

Nicolis G., Prigogine I. Self-Organization in Nonequilibrium Systems, Wiley-Interscience, New York, 1977, ISBN 0-471-02401-5

Ortoleva P. Geochemical self-organization, Oxford Monographs on Geology and Geophysics (1993).

Pertsov A. M., Vinson M., Müller S. C. Three-dimensional reconstruction of organizing centers in excitable chemical media. Physica D 63, 233 (1993).

Schlicht L., Spilgies J.-H., Runge F., Lipgens S., Boye S., Schübel D., Ilgenfritz G. Temperature-, electric field- and solute-induced percolation in water-in-oil microemulsions, Biophys. Chem. 58, $1-2,(1996)$.

Schmidt B., DeKepper P., Müller S. C. Destabilization of Turing structures by electric fields, Phys. Rev. Lett. 90 (118302), 1-4 (2003).

Schütze J., Steinbock O., Müller S. C. Forced vortex interaction and annihilation in an active medium, Nature 356, 45-47 (1992).

Schwartz L. J., DeCiantis C. L., Chapman S., Kelley B. K., Hornak J. P. Motions of water, decane, and bis(2-ethylhexyl)sulfosuccinate sodium salt in reverse micelle solutions, Langmuir 15, 5461 (1999).

Ševčiková H., Marek M. Chemical front waves in an electric field, Physica D 13, 379 (1984).

Ševčiková H., Marek M., Müller S. C. The reversal and splitting of waves in an excitable medium caused by an electrical field, Science 257, 951-954 (1992).

Ševčíková H., Schreiber I., Marek M. Dynamics of oxidation Belousov-Zhabotinsky waves in an electric field, J. Phys. Chem. 100, 19153 (1996).

Steinbock O., Müller S. C. Control of spiral waves in excitable media by external perturbation // Handbook of Chaos Control (H. G. Schuster, Ed.), Wiley-VCH, Weinheim, New York, 591614 (1999).

Steinbock O., Schütze J., Müller S. C. Electric-field-induced drift and deformation of spiral waves in an excitable medium, Phys. Rev. Lett. 68, 248-251 (1992).

Tyson J. J. The Belousov-Zhabotinskii reaction, Lect. Notes in Biomath. 10, Springer, Berlin (1976).

Winfree A. T. When time breaks down, Princeton University Press, New Jersey (1987). 